\title{
Head direction maps remain stable despite grid map fragmentation
}

\author{
Jonathan R. Whitlock ${ }^{1}$ and Dori Derdikman ${ }^{2 *}$ \\ Kavli Institute for Systems Neuroscience and the Centre for the Biology of Memory, Norwegian University of Science and Technology, Trondheim, Norway \\ 2 Rappaport Faculty of Medicine, Technion - Israeli Institute of Technology, Haifa, Israel
}

\section{Edited by:}

Lisa M. Giocomo, Norwegian

University of Science and

Technology, Norway

Reviewed by:

John L. Kubie, The State University

of New York, USA

Benjamin J. Clark, University of

Lethbridge, Canada

\section{*Correspondence:}

Dori Derdikman, Rappaport Faculty of Medicine, Technion - Israel Institute of Technology, Bat Galim, Haifa 31096, Israel. e-mail: derdik@technion.ac.il
Areas encoding space in the brain contain both representations of position (place cells and grid cells) and representations of azimuth (head direction cells). Previous studies have already suggested that although grid cells and head direction cells reside in the same brain areas, the calculation of head direction is not dependent on the calculation of position. Here we demonstrate that realignment of grid cells does not affect head direction tuning. We analyzed head direction cell data collected while rats performed a foraging task in a multi-compartment environment (the hairpin maze) vs. an open-field environment, demonstrating that the tuning of head direction cells did not change when the environment was divided into multiple sub-compartments, in the hairpin maze. On the other hand, as we have shown previously (Derdikman et al., 2009), the hexagonal firing pattern expressed by grid cells in the open-field broke down into repeating patterns in similar alleys when rats traversed the multi-compartment hairpin maze. The grid-like firing of conjunctive cells, which express both grid properties and head direction properties in the open-field, showed a selective fragmentation of grid-like firing properties in the hairpin maze, while the head directionality property of the same cells remained unaltered. These findings demonstrate that head direction is not affected during the restructuring of grid cell firing fields as a rat actively moves between compartments, thus strengthening the claim that the head direction system is upstream from or parallel to the grid-place system.

Keywords: entorhinal cortex, head direction cells, grid cells, hippocampus, remapping, cognitive map

\section{INTRODUCTION}

The ancient Greek philosopher Heraclitus observed thousands of years ago that everything flows ( $\Pi \alpha \dot{\nu} \tau \alpha \grave{\rho} \varepsilon \tilde{\imath})$. The world is constantly changing, and no two sensory stimuli are totally alike. In order to accommodate to the perpetual change of the world, the brain must extract invariant aspects of stimuli it senses (Gibson, 1979). Two examples of such invariants which can be extracted are place and head direction. It is thus not too surprising that the brain has evolved two separate systems: one for the representation of self-location, and another for the representation of head direction. We will overview the two systems below and then describe an experiment demonstrating their separability.

\section{SPATIAL REPRESENTATION IN THE HIPPOCAMPUS AND ENTORHINAL CORTEX}

Our understanding of the neural representation of spatial location began with the discovery of "place cells" in area CA1 of the hippocampus in 1971 (O'Keefe and Dostrovsky, 1971). As their name suggests, place cells were found to exhibit the striking behavioral correlate of discharging selectively when rats occupied a particular location in a recording arena. The remarkable spatial specificity and stability exhibited by place cells suggest that they are key elements of the brain's spatial memory system (see O'Keefe, 2007 for review), and may provide the neural substrate for a "cognitive map" which provides "an objective spatial framework within which the items and events of an organism's experience are located and interrelated" (Tolman, 1948; O'Keefe and Nadel, 1978).

Studies in the decades since the discovery of place cells, however, suggest that spatial representations in the hippocampus are neither immutable nor absolute. Some of the first evidence for this came with the observation that place cells fire at different locations and at different firing rates in response to changes in visual cues or in the shape of the recording arena (Muller and Kubie, 1987; Bostock et al., 1991). Later termed hippocampal "remapping," this phenomenon can take one of two forms in the spatial domain - "rate remapping" and "global remapping" (Leutgeb et al., 2005). Rate remapping refers to a condition wherein place cells change their firing rate but maintain the same spatial location and has been shown to occur following nonspatial manipulations of the environment (e.g., reversing the colors of the walls and cue card in a recording arena) (Leutgeb et al., 2005) (Figure 1A). Global remapping, in which the combination of co-active cells changes and place fields undergo a complete reorganization of firing locations and firing rates, occurs following more profound changes in spatial inputs such as changing recording rooms or substantially altering the features of a recording arena (Leutgeb et al., 2005; Wills et al., 2005; Jezek et al., 2011) (Figure 1B).

A precise and more generalized spatial code was recently discovered one synapse upstream of the hippocampus in the form of grid cells in the medial entorhinal cortex (MEC) (Hafting et al., 
A

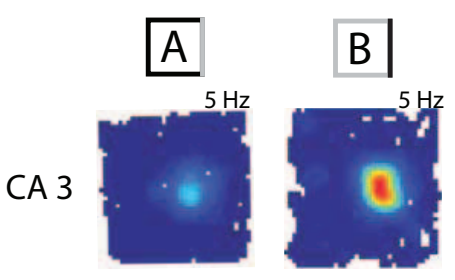

B

$A$

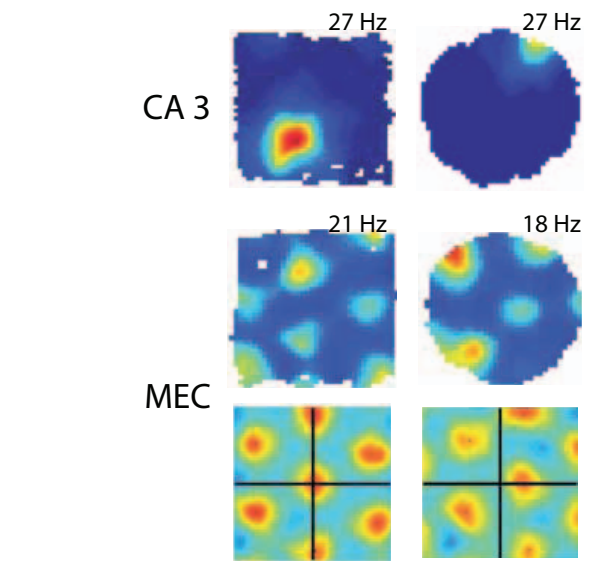

(B)

C
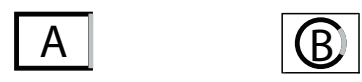

PrS
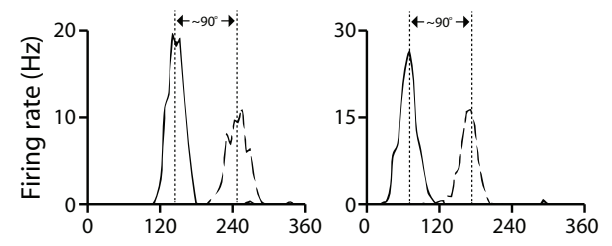

Head direction (degrees)

FIGURE 1 | Remapping experiments have shown that spatial maps in hippocampus and MEC are coordinated, while head direction preferences are coordinated across cells in presubiculum. (A) An example of rate remapping in area CA3 of the hippocampus. During rate remapping, place fields maintain a constant position but change firing rate; grid maps do not change during rate remapping. Left: Rate map of a CA3 place cell when the rat is in a box with three black walls and one white wall. Right: The same cell has a higher firing rate when the color scheme of the walls is reversed. (B) Switching recording enclosures elicits global remapping in the hippocampus, in which place cells change both their firing rate and position, and causes gird cells to undergo a shift in X-Y spatial phase that is apparent in the spatial cross-correlations beneath the rate maps [the cross-correlations are a comparison of rate maps at all overlapping spatial shifts for the grid cell in environment A vs. A' (left) or in A vs. B (right)] (adapted from Fyhn et al., 2007). (C) Changing recording enclosures causes head direction cells in the dorsal presubiculum (PrS) to change their preferred directions, but the relative difference in the cells' preferred directions is conserved across environments. Left: Head direction tuning for a pair of cells recorded from PrS in a rectangle. Right: the cells shift their directional tuning when the rat is placed in a cylinder, but the relationship between the cells' preferred firing directions is nearly the same (adapted from Taube et al., 1990b).
2005). Unlike place cells, grid cells have multiple firing fields arranged in an iterative triangular array which covers the entire environment explored by a rat, and the same stereotypic pattern is expressed irrespective of local landmarks and non-spatial cues (Fyhn et al., 2007; Solstad et al., 2008). Although grid cells express similar hexagonal firing patterns in different open recording arenas, switching recording enclosures was shown to cause grid maps to shift their X-Y spatial phase and to rotate to a new orientation (Fyhn et al., 2007) (Figure 1B, bottom).

Recent studies comparing simultaneously recorded place cells and grid cells suggest that spatial maps in the hippocampus and MEC may be linked causally. In the study of Fyhn et al., 2007, for example, it was shown that global remapping (but not rate remapping) in the hippocampus was always attended by the reorientation and realignment of grid maps in MEC. Later work showed that place cells and grid cells underwent a comparable restructuring of their firing fields when animals were moved from an open recording arena into a compartmentalized maze consisting of a series of interconnected alleys (i.e., a hairpin maze; Figure 2A) (Derdikman et al., 2009). It was found that the hexagonal structure of grid cells fragmented into a series of submaps that repeated across alleys in which the animals ran in the same direction, and that the firing fields of place cells repeated across alternating alleys in a similar manner. It was concluded that spatial maps in both areas were transformed into repeating submaps which reset at the turning point from one alley to the next (Derdikman et al., 2009; Derdikman and Moser, 2010). Together, these findings support the view that changes in the configuration of spatial maps in one area are attended by changes in the maps expressed in the other area, suggesting that the hippocampus and MEC comprise an integrated circuit within which self-location signals are generated.

\section{HEAD DIRECTION REPRESENTATION IN THE MAMMALIAN BRAIN}

Head direction cells, which discharge when an animal is facing a particular direction irrespective of spatial location, were first reported in the 1980s by Ranck (1985), and have since been observed in many different brain regions, including the anterior dorsal thalamus (Taube, 1995), the dorsal presubiculum (Taube et al., 1990a; Boccara et al., 2010), retrosplenial cortex (Chen et al., 1994) and MEC (Sargolini et al., 2006). All known areas which contain grid cells also contain head direction cells (Sargolini et al., 2006; Boccara et al., 2010). Furthermore, it has recently been shown that there are orderly anatomical connections between patches of head direction cells and patches of putative grid cells in the MEC (Burgalossi et al., 2011). Consistent with the anatomical comingling of these cell types, there is evidence that grid cell maps and head direction signals in MEC are linked in that both follow the rotation of visual landmarks as a coherent ensemble (Solstad et al., 2008). There is, however, a substantial literature supporting the notion that head direction signals may be computed upstream from cells encoding spatial location, and that the two types of representations can change at least partly in parallel with one another.

Shortly after the discovery of head direction cells, Taube and colleagues demonstrated that head direction cells respond differently than place cells in circumstances which cause hippocampal 


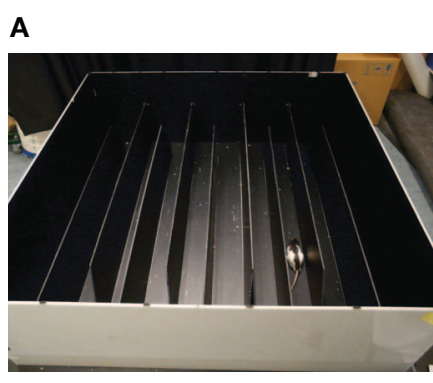

C
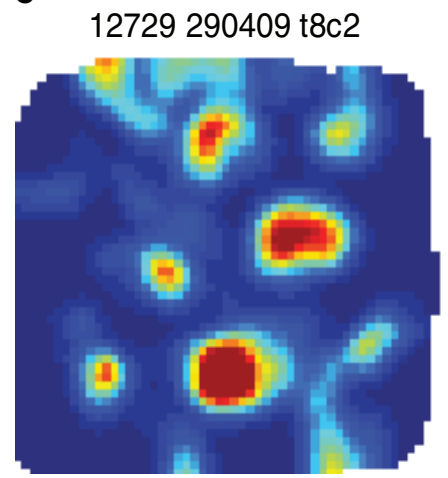

E

Outbound

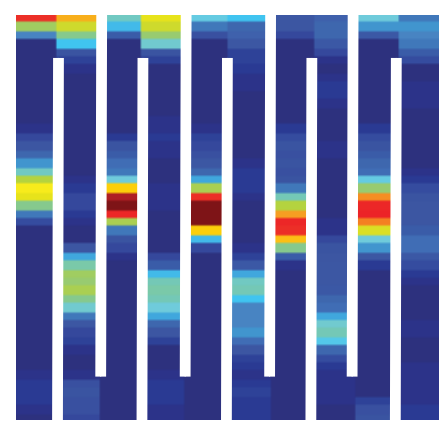

G

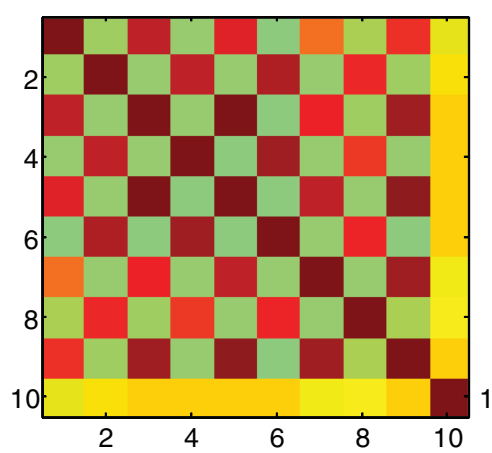

FIGURE 2 | Break-down of grid cells in the hairpin maze. (A) The hairpin maze setup, as used in the Derdikman et al. (2009): the rat ran first in the open-field, then in the hairpin maze, and then finally in the open-field again. (B) Grid score histogram (value averaged for first and second open-field sessions) for all 61 cells. Chosen threshold for conjunctive cells (0.5) is marked in red. (C-F) Example of a conjunctive cell when the rat is in the open-field (C), when the rat is running

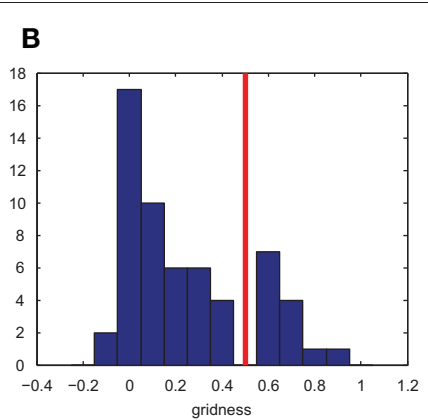

D

\section{$4.2226 \mathrm{~Hz}$}

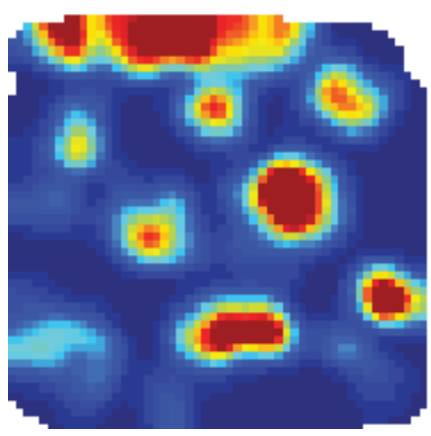

$\mathbf{F}$

Inbound

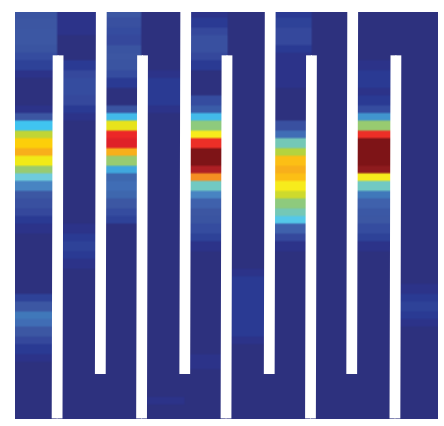

H

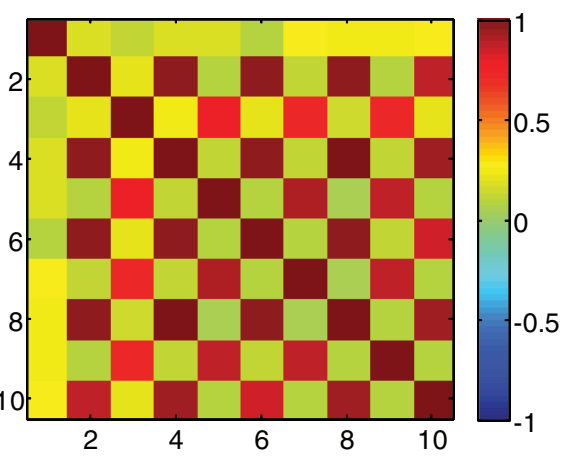

outbound on the hairpin maze (E), when it is running inbound in the same maze (F) and when it is put for a second time into the open-field (D). (G,H) Correlation matrix between rate maps from the population of conjunctive cells in this study $(N=13)$ during outbound (G) and inbound (H) runs. The checkboard pattern results from the higher correlation between every second arm for both inbound and outbound runs. 
remapping (Taube et al., 1990b). Namely, it was found that moving an animal from one arena to another caused simultaneously recorded head direction cells to shift to new directions, but that the angular distance between the cells' preferred directions was conserved in the different environments (i.e., the cells remained in register, Figure 1C). Subsequent studies showed that place cells could undergo partial spatial remapping independently of head direction cells in the anterior thalamus (Knierim et al., 1995), and that head direction signals and grid maps rotate arbitrarily with regard to one another in different recording rooms (Solstad et al., 2008). More recently it was found that inactivation of the medial septum caused a loss of the spatial structure of grid cells in the MEC while head direction cells were unaffected (Brandon et al., 2011; Koenig et al., 2011), suggesting that head direction cells do not depend on grid cells for their directional selectivity. This idea is further supported by developmental studies in rats showing that head direction signals are already mature before place cells and grid cells begin to show adult-like specificity (Langston et al., 2010; Wills et al., 2010).

In the current study we provide new analyses of data from head direction cells and grid cells published previously by Derdikman et al. (2009). In a subset of experiments from that study we monitored head direction of rats in both the open-field and the hairpin maze and found that head direction signals did not change between the tasks despite the total restructuring of grid maps in MEC. Our findings strengthen the argument that head direction signals are expressed upstream from spatial maps, such that representations of spatial location can change without affecting representations of head direction.

\section{MATERIALS AND METHODS}

As this paper performs analysis of previously collected data, behavioral, and electrophysiological methods for these experiments were described in more detail in Derdikman et al. (2009). In short, neuronal activity was recorded from MEC in five male Long-Evans rats (3-5 months old, 350-450 g at implantation and testing), using an Axona data-acquisition system. Tetrodes were inserted above the dorsocaudal part of MEC. Rats collected crumbs of chocolate cereal thrown randomly into a black 1.5$\times 1.5-\times 0.5-\mathrm{m}$ open-field arena surrounded by a black curtain. A white cue card $(95 \times 45 \mathrm{~cm})$ was placed on the curtain $110 \mathrm{~cm}$ above the floor. Training in the hairpin maze began when the rat regularly covered the entire open-field on a 20 -min trial. Nine opaque Perspex walls were inserted into parallel grooves carved into the underlying floor. The walls were $135 \mathrm{~cm} \times 30 \mathrm{~cm} \times 1 \mathrm{~cm}$. The rats were trained to run from east to west (outbound) and from west to east (inbound) on alternating trials without interruption. During testing, the rats ran for 20-min in the open-field, followed by two 20 -min runs in the hairpin maze, followed by another 20-min run in the open-field. Between runs, the rats rested in a flower pot next to the maze or in their home cage.

Spike sorting was performed offline using graphical clustercutting software. Position estimates were based on tracking of one LED on the headstage. The tracked positions were smoothed offline with a 15-point mean filter. Tracking errors were removed offline by an interactive MATLAB script that assumed that the path did not cross walls. The position data were sorted into
$1 \times 1 \mathrm{~cm}$ bins and the firing rate was determined for each bin in the open-field and in the hairpin maze. Following the experiments, the rats underwent perfusion, histological sectioning, and Nissl staining, in order to determine the electrode positions.

In the current paper we used the subset of the data from Derdikman et al. (2009) in which we tracked the position of two LEDs in the hairpin maze ( $N=5$ rats), so that we could derive the head direction of the rat both in the open-field and in the hairpin maze.

The grid score of the cells was calculated using a rotationalsymmetry score (Sargolini et al., 2006; Boccara et al., 2010; Langston et al., 2010). Briefly, spatial autocorrelation maps were calculated for each rate map. The degree of spatial periodicity (gridness) was determined for each recorded cell by taking a circular sample of the autocorrelogram, centered on the central peak, and comparing rotated versions of this sample. The Pearson correlation of this circle with its rotation in $\alpha$ degrees was obtained for angles of $60^{\circ}$ and $120^{\circ}$ on one side and $30^{\circ}, 90^{\circ}$, and $150^{\circ}$ on the other. "Gridness" was defined as the minimum difference between any of the elements in the first group and any of the elements in the second. For each cell the grid score calculation was made on multiple circular samples surrounding the center of the autocorrelogram, with circle radii increasing in steps of $5 \mathrm{~cm}$ from $20 \mathrm{~cm}$, up to the width of the box. Gridness was defined as the best score from these successive samples. The distribution of grid scores (averaged over both open-field sessions) in our study was bi-modal (Figure 2B), and thus we chose the gridness threshold at the trough of the bi-modal distribution (Gridness $>0.5$ ).

In order to calculate the preferred head direction for each cell, we constructed a head direction histogram for each cell, and then calculated the direction and length of the Rayleigh vector for each head direction histogram (Boccara et al., 2010). In the Head direction histogram calculations we used bins of $6^{\circ}$ without smoothing for display purposes (Figure 3), and $30^{\circ}$ without smoothing for the population analyses. We note that the use of different bins for head direction calculation did not have a substantial effect on head direction estimates (derived from the angle of the Rayleigh vector), apart for the cases of very low firing rates. Data analysis was carried out using MATLAB. The $p$-value of correlation coefficients was computed by transforming the correlation to create a $t$ statistic having $n-2$ degrees of freedom. Each instance of monte-carlo shuffling of head direction data was done by permuting the order of the cells in one condition when comparing it to the other condition.

\section{RESULTS}

As described in Derdikman et al. (2009), the rats were first trained to run randomly in an open-field, $1.5-\times 1.5-\mathrm{m}$ box. When the rats had covered the entire arena repeatedly across trials, they were trained to run in a multi-compartment hairpin maze constructed from Perspex walls inserted into the open-field box (Figure 2A). The rats ran outbound (west) and inbound (east) in an alternating manner. Daily sessions consisted of a 20-min trial in the openfield, two 20-min trials in the hairpin maze and a second 20-min trial in the open-field. Recording electrodes were placed in the MEC. In Derdikman et al. (2009) we found that grid cells formed a discrete spatial representation for each sub-environment when 

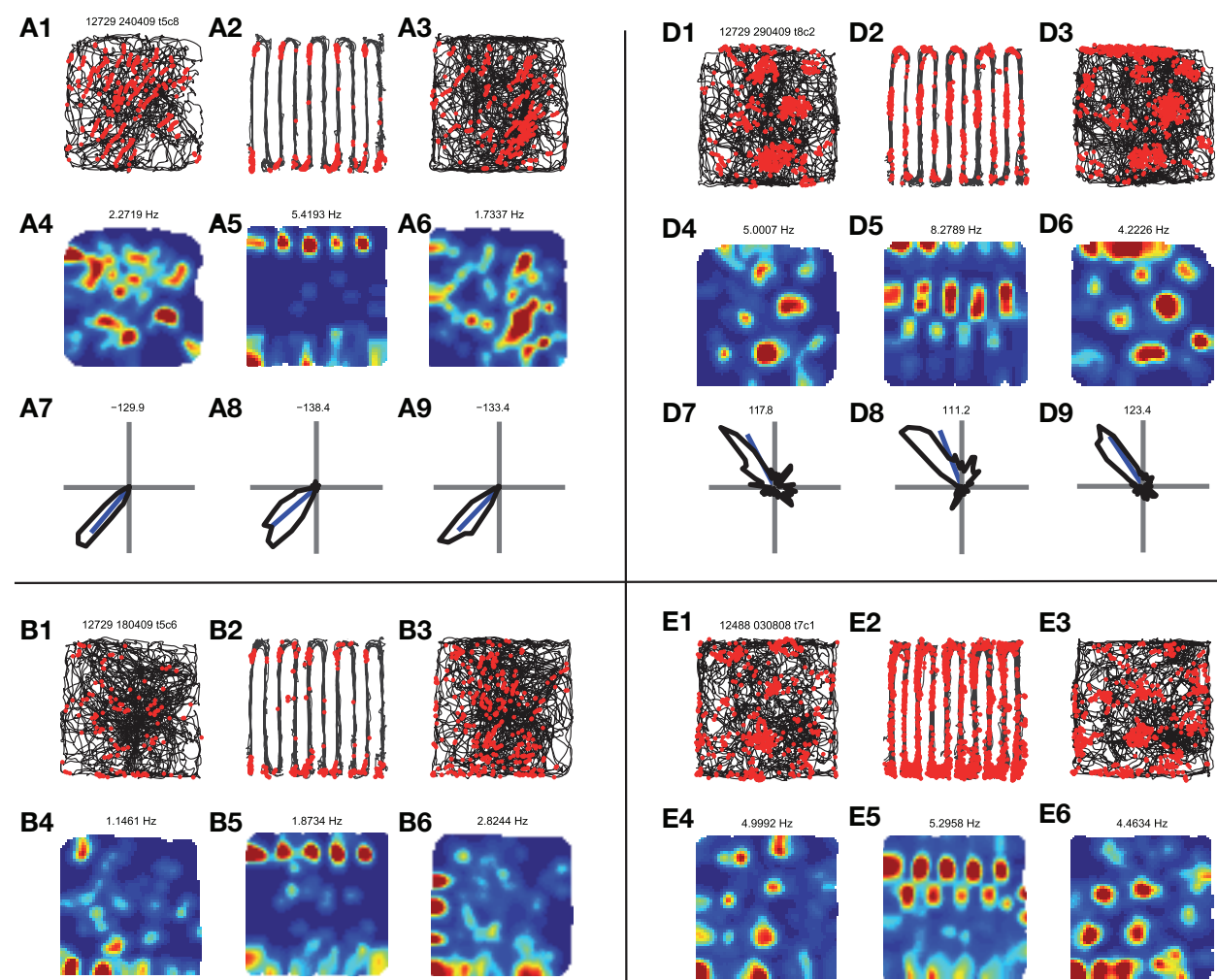

B7

B8

B9
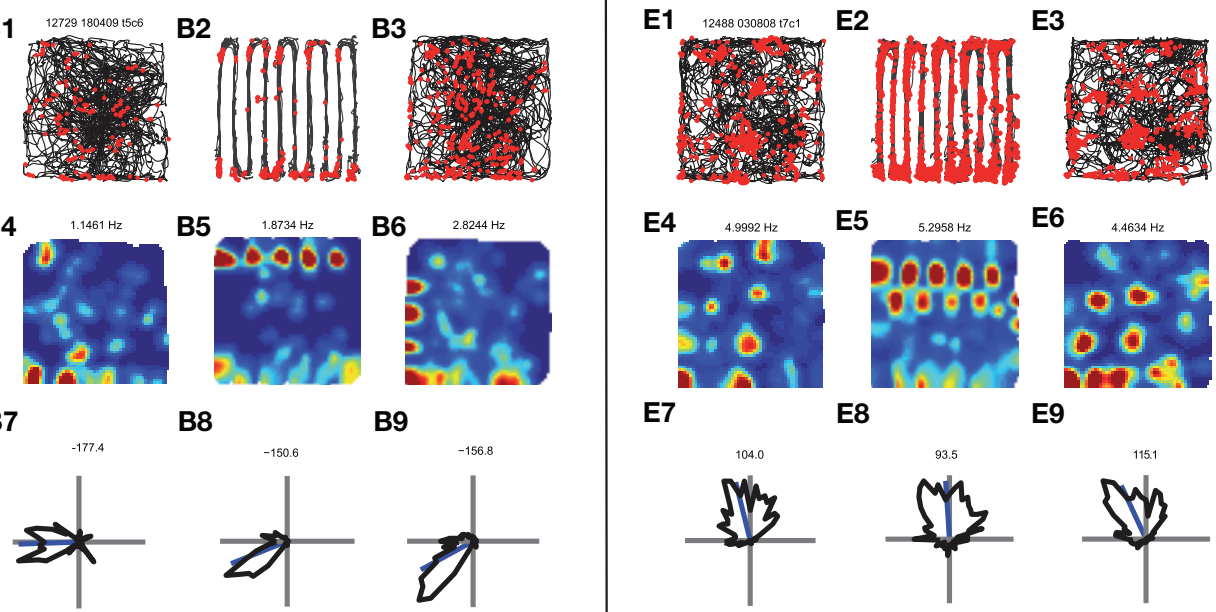

E4

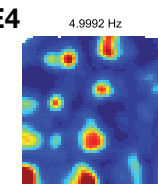

E5

E6

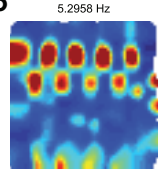

$4.4634 \mathrm{~Hz}$

E7

E8
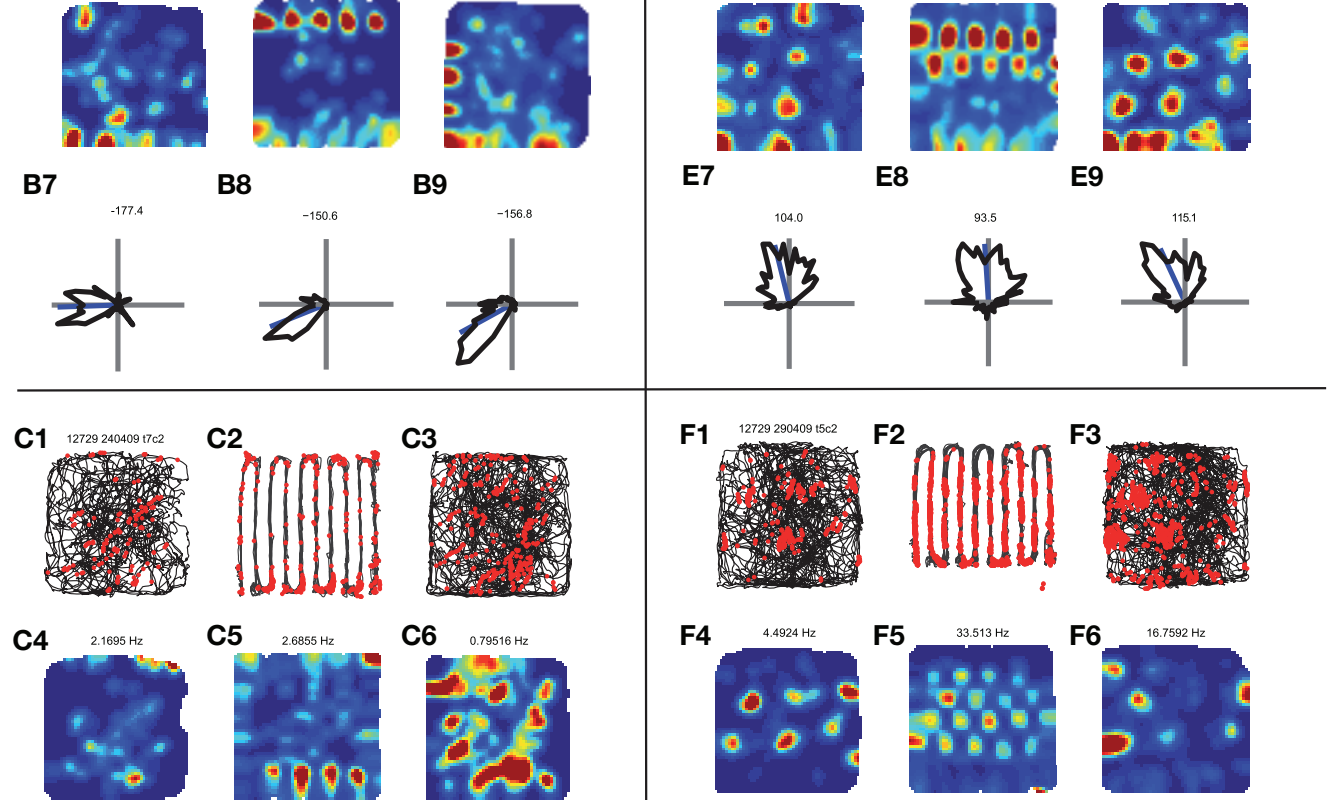

C7

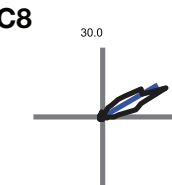

C9

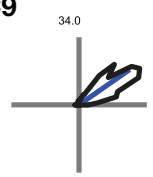

FIGURE 3 | Comparison of the behavior of head direction cells in the open-field vs. the hairpin maze. (A) A head direction cell. (A1) Path and spikes in first open-field trial. (A2) Path and spikes in hairpin trial (A3) path and spikes in second open-field trial. (A4) Rate map in first open-field trial (generated from A1). (A5) rate map in hairpin trial (generated from A2). (A6) Rate map in second open-field trial (generated from A3). (A7) Head direction polar histogram in first open-field. Blue line is the preferred direction of the cell. (A8) Head direction polar histrogram in hairpin trial. (A9) head direction polar histogram in second open-field. Note the similar

F4

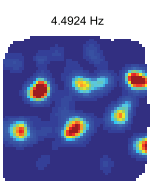

F7

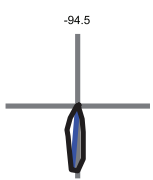

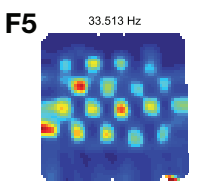

F8

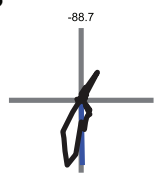

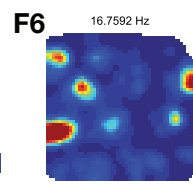

F9

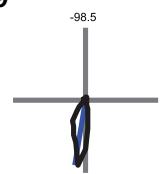

head direction between the open-field and hairpin trials. (B,C) Two additional examples of head direction cells. Panels organized as in $\mathbf{A}$ (D,E,F) Examples of the behavior of conjunctive $\{$ head direction $\times$ grid $\}$ cells. Note that for all of these examples the grid pattern seen in the open-fields (D4,D6,E4,E6,F4,F6) breaks down inside the hairpin maze (D5,E5,F5), and repetitive patterns are seen between arms, as described in Derdikman et al. (2009), and in Figure 2 above. However, head direction remains constant between the open-field conditions (D7,D9,E7,E9,F7,F9) and the hairpin condition (D8,E8,F8). 
the open environment was divided into multiple compartments. There was no master grid spanning across the alleys of the hairpin maze; instead the grid cell representation reset sharply each time the rat turned from one compartment to the next.

We extracted from the data 61 head direction cells (Rayleigh vector length $>0.25$ in at least one of the open-field conditions), out of which 13 were clearly conjunctive head direction $\times$ grid cells (grid score $>0.5$, Figure $2 \mathbf{B}$ ), and wanted to check what transformation the grid pattern and head direction signal underwent between the open-field and the hairpin maze. As demonstrated already in Derdikman et al. (2009), the hexagonal firing pattern seen in grid cells in the open-field broke down within the hairpin maze (Figures 2C-F). Furthermore, also on this subset of the data, every second alley correlated in the hairpin, and the firing was different during outbound and during inbound runs of the rat, creating a check-board pattern when comparing arm-toarm rate correlations, similar to the check-board patterns found in Derdikman et al. (2009) (Figures 2G,H).

Single examples of head direction cells (Figures 3A-C) demonstrated that the preferred direction of the cells was quite constant between the open-field and hairpin conditions. For example, one cell had a preferred direction of $-129.9^{\circ}$ in the first open-field condition (Figure 3A7), a preferred direction of $-138.4^{\circ}$ in the hairpin maze (Figure 3A8), and a preferred direction of $-133.4^{\circ}$ in the second open-field condition (Figure 3A9). Similar results were seen in other head direction cells as well (Figures 3B,C). Furthermore, for a cell that had conjunctive grid $\times$ head direction properties (Figures 3D-F), the grid pattern broke-up in the hairpin maze (compare Figures 3D4-D6) while the preferred head direction remained quite constant between conditions (Figures 3D7-D9). This phenomenon repeated itself in additional conjunctive cell examples (Figures 3E,F).

We wished to quantify this phenomenon across the whole head direction and conjunctive cell population in our data. We compared the preferred head direction of the cells in the different conditions (Figures 4A-D). As expected, the head direction cells did not change their preferred direction much between the first and second open-field conditions. The median absolute value of angle change was $9.2^{\circ}$, (Figure 4C) which was lower than 100,000 runs of shuffled data (i.e., $p<10^{-5}$; see Methods). Next, we compared the preferred direction of the cells between the first open-field and the hairpin conditions. Also in this case, the preferred head direction between the first open-field and hairpin did not change much. The median absolute value of angle change was $8.8^{\circ}$ (Figure 4D), which was lower than 100,000 runs of shuffled head direction data (Figure 4E). Furthermore, the head direction angle of each cell did not change significantly between different arms (Figures $\mathbf{4 F}-\mathbf{H}$ ). The median difference between the head direction angle in a specified arm of the hairpin maze and the mean head direction angle for all arms together was $11.97^{\circ}$ for all cells (Figure 4G), which was lower than 100,000 runs of shuffled data (e.g., Figure $4 \mathbf{H}$ ). Thus we found that the preferred head direction did not change significantly between the open-field and hairpin conditions, and was similar in different subcompartments within the hairpin maze.

In Derdikman et al. (2009), the grid cells broke-up in the hairpin-maze. These results were also reproduced in the subset of conjunctive grid $\times$ head direction cells used in this study. Cells with high grid scores ( $\geq 0.5, N=13$; Figure 4I, red stars) had highly correlated rate maps between the first and second openfield conditions (mean $r=0.535 \pm 0.049$ ), but failed to maintain a similar spatial correlation between the open-field and hairpin rate maps (mean $r=0.081 \pm 0.035$ ), implying that, as expected from Derdikman et al. (2009), the conjunctive cells did not have a grid representation in the hairpin maze. Thus, spatial representations broke-up between the open-field and the hairpin maze in this cell sample as well (similar to the entire cell sample, reported in Derdikman et al., 2009). However, the subset of conjunctive cells did not change their preferred head direction from the open-field to the hairpin maze. The median absolute value of angle change for conjunctive cells was $11.7^{\circ}$, which was higher than only 17 instances out of 100,000 runs of shuffled data (i.e., $p \approx 0.00017$ ).

We conclude that while the grid pattern disintegrated in the hairpin maze, both head direction cells and conjunctive grid cells did not change their preferred head direction between the different conditions.

\section{DISCUSSION}

In this paper we demonstrate that head direction cells and conjunctive head direction $\times$ grid cells conserve their preferred head direction between the open-field and hairpin maze despite the fragmentation of grid cell maps, and that the head direction signal within the hairpin maze remains constant across individual alleys despite the alternation between distinct submaps in the MEC. These findings show that the representation of head direction is unaffected by the restructuring of grid cell spatial maps and imply that head direction may be computed upstream of spatial location signals.

\section{COMPARISON WITH PRIOR STUDIES}

The first set of data in this study show that head direction signals in MEC were the same regardless of whether rats ran in the open-field or the hairpin maze. This invariance of head direction representation with regard to the spatial layout of the tasks suggests that the animals oriented themselves using either idiothetic cues or visual landmarks outside the arena (in this case, a large white cue card was suspended above the south wall), and speaks against the view that heading orientation is derived purely from environmental geometry (Cheng, 1986). Rather, our observations are in line with recent studies showing that head direction signals in a variety of brain areas are unaffected by changes in environmental geometry, either in differently shaped recording enclosures (Knight et al., 2011) or as rats moved from one compartment to the next in a 14-unit T-maze (Yoder et al., 2011). These studies and ours support the view that idiothetic cues, distal visual landmarks or the combination of both play a primary influence in setting an animal's sense of orientation in environments with differing geometries (see also Goodridge and Taube, 1995; Blair, 1996).

Despite the stability of the head direction signal in MEC between the open-field and hairpin maze, the compartmentalization of the environment caused hexagonal grid maps to fragment into non grid-like submaps (Derdikman et al., 2009). 

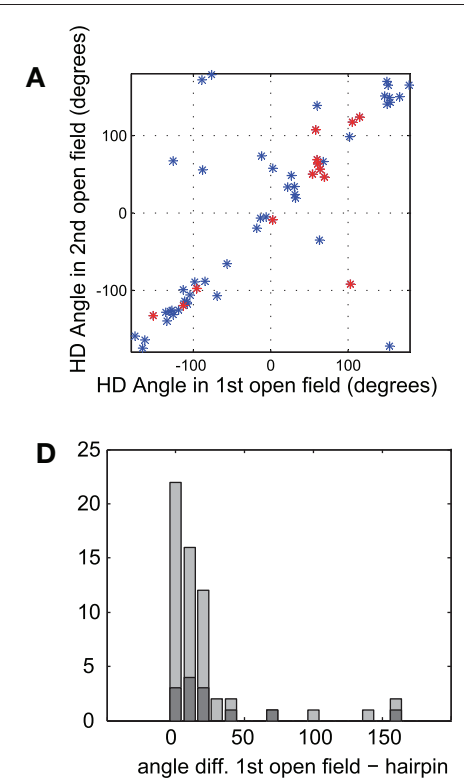

G

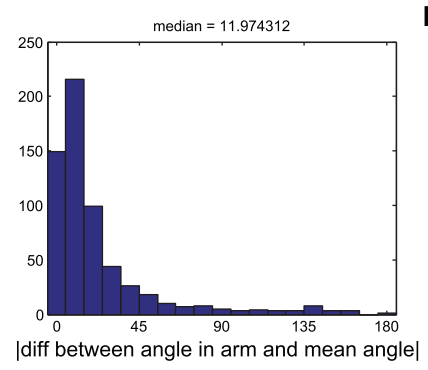

B

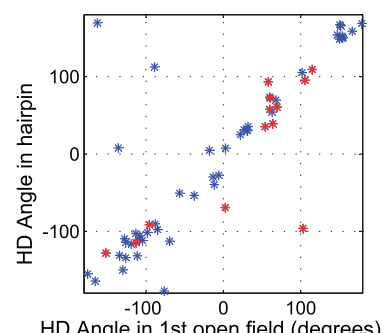

HD Angle in 1st open field (degrees)

E

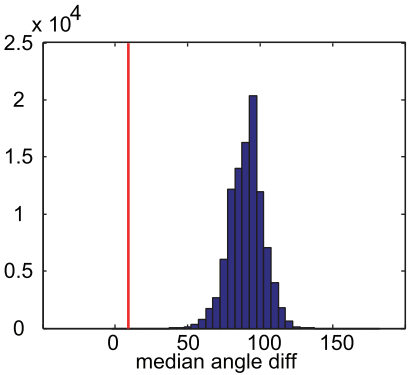

H

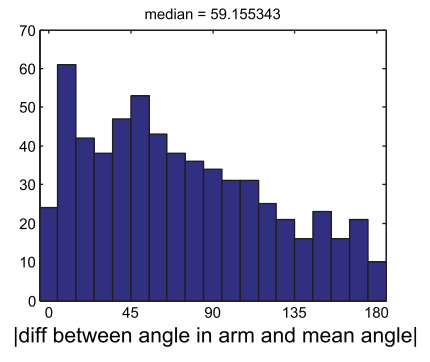

C

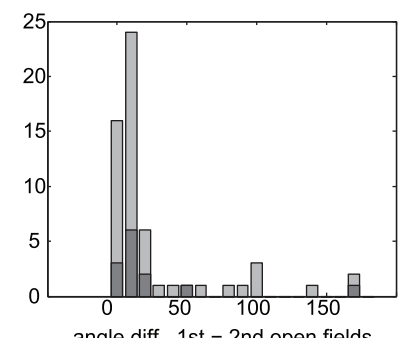

angle diff. 1 st -2 nd open fields
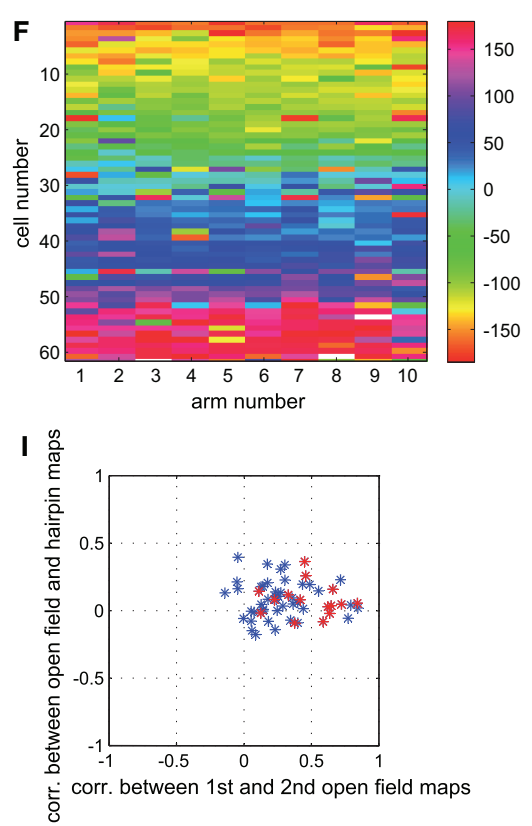

FIGURE 4 | Head direction is similar for all conditions. (A) Head direction angle preference for each cell, calculated from the direction of the Rayleigh vector, in the first vs. second open-field conditions.

Red points mark sub-populations of conjunctive grid $\times$ head direction cells (gridness $>0.5$ in open-fields). (B) Head direction angle preference in first open-field vs. hairpin maze. Red points as in A. (C) Histogram of absolute angle differences between head direction preferences in first and second open-field conditions. Dark gray bars mark conjunctive cells. Absolute angle difference can range between $0^{\circ}$ and $180^{\circ}$, with an expected median of $90^{\circ}$, as can be seen in shuffled data (panel E). (D) Histogram of absolute angle differences between head direction preferences in first open-field condition vs. hairpin condition. Dark gray bars mark cells with high grid scores. (E) Real value of median angle difference between head direction in open-field vs. hairpin maze (shown in $\mathbf{E}$ ) was $8.8^{\circ}$, lower than 100,000 monte-carlo angle differences generated from shuffled data. (F) Color-coded matrix of head direction preferences of each cell in each arm of the hairpin-maze. Cells are ordered according to the circular mean of their preferred head direction angle. (G) Histogram of absolute value of difference between head direction in each arm and mean head direction in all arms for all cells. The median difference between the head direction preference in a single arm and the mean for all arms is $11.97^{\circ} \mathbf{( H )}$ Similar histogram as in $\mathbf{G}$, but with shuffled head directions (I) Comparison of spatial correlation between the two open-field rate maps (such as in Figure 3, A4 correlated with A6) vs. correlation between open-field rate maps and rate maps generated from hairpin (such as in Figure 3, A4 correlated with A5). Conjunctive cells (red points) show a higher correlation $(x$-axis) between two open-field conditions than between open-field and hairpin conditions (y-axis), demonstrating the breakup of the map in the hairpin maze, as described more thoroughly in Derdikman et al. (2009). However, the very same cells (dark gray bars in panels $\mathbf{C , D}$ ) show strikingly similar head direction preferences between hairpin and open-field conditions.
This observation demonstrates that head direction tuning is expressed upstream from spatial signals in MEC, and is consistent with anatomical data suggesting that head direction cells in the deeper layers of MEC serve as inputs to grid cells in superficial layers (Canto et al., 2008). Though input from head direction cells may play a critical role in the etiology of grid cell firing patterns, as posited by oscillatory-interference models (Burgess et al., 2007) and attractor-network models (Fuhs and Touretzky, 2006; McNaughton et al., 2006) of grid map formation, the profound structural transformation of grid maps between the open-field and the hairpin maze (despite the unchanging directional signals) suggests that other inputs must also be at play in determining the geometric organization of grid maps.

We also found that head direction cells in MEC did not change within the hairpin maze when the rats passed from one compartment to the next, or between east- and westbound trajectories. This result contrasts somewhat with earlier studies showing that head direction cells change firing preferences readily in different recording enclosures (Taube et al., 1990b). A key methodological feature of the Taube et al. study, however, was that the animals 
were passively transported between the two recording environments, allowing the subjects to become disoriented and for the head direction cells to change their preferred azimuth to a different one. It is now appreciated that head direction signals are maintained across different environments when animals are able to move actively between them, even when one of the recording enclosures is novel (Taube and Burton, 1995; Stackman et al., 2003), or when the arenas are connected but in separate rooms (Yoder et al., 2011). Thus, the continuity of self-motion signals, presumably related to path integration, is sufficient to anchor the frame of reference for the head direction system even when the pattern of sensory inputs has changed completely. Such a mechanism would allow the rats in the present study to maintain a constant representation of head direction in the hairpin maze since the animals maintained continuous motor control over their trajectory.

Given that head direction cells maintained a similar heading throughout the hairpin maze, it is again striking that spatial submaps expressed by nearby grid cells and conjunctive cells were different depending on north-south running direction and during east- and westbound traversals. In the absence of changes in directional inputs, the alternating firing patterns we observed may have arisen due to a combination of idiothetic cues and differences in the precise visual inputs available to the animals as they made north- and southward laps. The causal relationship between representations in grid cells and head direction could perhaps be further elucidated if head direction signals were re-anchored to a new set of visual landmarks between recording sessions in the hairpin maze, and it could then be determined whether the grid cell submaps showed a similar realignment. Recordings in the dark would also help isolate the precise contribution of path-integration cues.

\section{INHERITANCE OF HEAD DIRECTION AND SPATIAL PROPERTIES FROM UPSTREAM AREAS}

The observation that head direction cells and grid cells in MEC behaved strikingly differently when the rats were transferred from the open-field to the hairpin maze demonstrates that spatial and directional signals can be disentangled from one another, even amongst co-localized cells. The fact that spatial maps in conjunctive cells were totally restructured while their directional tuning remained unchanged demonstrates that the two types of information can be computed separately, and may imply that the neural signals used to compute head direction and spatial location are conveyed from independent upstream pathways. For instance, it is known that conjunctive cells in MEC receive direct and indirect spatial input from place cells in the hippocampus as well as grid cells in MEC, presubiculum, and parasubiculum (Canto et al., 2008; Boccara et al., 2010). Precise spatial tuning in these structures, in turn, likely depends on visuo-spatial and self-motion signals from cortical afferents which include postrhinal, parietal, and retrosplenial areas (Burwell et al., 1998; Jones and Witter, 2007). The head direction signal, on the other hand, is most likely dependent on robust input from head direction cells in the presubiculum and parasubiculum, and may also depend partly on input from retrosplenial cortex (van Groen and Michael Wyss, 1990; Jones and Witter, 2007; Boccara et al., 2010; Canto and
Witter, 2010). Directional tuning in the presubiculum itself sits atop a hierarchy of subcortical relays which propagate heading signals that originate primarily in the medial vestibular nuclei (see Taube, 2007, for review).

While lesioning or inactivating the vestibular apparatus results in a profound impairment in head direction representation (Stackman and Taube, 1997; Stackman et al., 2002), lesions to more downstream areas such as retrosplenial cortex or the presubiculum cause more specific impairments in the stability or landmark control of otherwise intact directional signals (Goodridge and Taube, 1997; Clark et al., 2010). In contrast, lesioning or inactivating areas outside of the head direction cell circuit, such as posterior parietal cortex, medial septum, or hippocampus has no effect on the expression of head direction signal in the anterior dorsal thalamus or in MEC, but causes a substantial disruption of the spatial structure of grid cells (Golob and Taube, 1997; Calton et al., 2008; Bonnevie et al., 2010; Whitlock et al., 2010; Brandon et al., 2011; Koenig et al., 2011). Furthermore, it seems that the head direction property is not related to the network mechanisms which supposedly can induce remapping in the hippocampal-entorhinal system (Samsonovich and McNaughton, 1997; McNaughton et al., 2006). Thus, the existing data point to the interpretation that head direction and spatial location signals each rely on contributions from several brain areas which constitute at least partially parallel systems. Ultimately, the head direction system may influence the gridplace system (Calton et al., 2003), but the data presented here and elsewhere (Clark and Taube, 2011) suggest that head direction signals are not affected by the grid-place system in return, and rather maintain a constant and robust reading of direction in all environments.

\section{GEDANKEN ARGUMENTS FOR THE INDEPENDENCE OF THE HEAD DIRECTION SYSTEM FROM THE PLACE SYSTEM}

The supposed separation between the two systems can be understood also from more general arguments. While many idiothetic sensory channels can give clues about both spatial position and head direction, we point out here that, in principle, head direction information should be easier to estimate from such inputs. Two major sources believed to influence path integration (although others exist) are vestibular inputs and optic flow. It is known that we can gain information about our self-movement from our vestibular sense, through the semicircular canals which transduce mostly rotational movements, and through the otolith organs, which transduce mostly linear accelerations, although the functions of the two organs may be mixed (Taube, 2007). But note a major difference: in order to derive the linear position from the vestibular sense, the brain needs to perform a double integration, from linear acceleration (transduced mostly by the otolith organs), to linear velocity and then from linear velocity to linear position. While in order to derive angular head direction from the vestibular sense, the brain needs to perform only a single integration, from angular velocity to angular direction. This is because angular velocity is transduced directly by sensing the centrifugal force (which, as known from physics, is proportional to velocity and not to acceleration), in the otolith 
organs and/or the semicircular canals. The necessity for double integration ( $\iint$ acceleration $\rightarrow \int$ velocity $\rightarrow$ position $)$ in the linear case $v s$. the need for only a single integration ( $\int$ angular velocity $\rightarrow$ angular position) in the angular case suggests that computing linear position from the vestibular sense is harder and more prone to noise than computing angular position. Thus, path integration mechanisms relying on the vestibular sense may be used more easily to derive angular position (Skaggs et al., 1995) than linear position (McNaughton et al., 1996; Samsonovich and McNaughton, 1997). It is, therefore, not too surprising that removing vestibular inputs to the head direction system has a strong effect on head direction cells (Stackman and Taube, 1997; Stackman et al., 2002) and, perhaps as a consequence, impairs path-integration abilities in a variety of species including cats, dogs, humans, and rodents (Beritoff, 1965; Mittelstaedt and Mittelstaedt, 1980).

A second important source of positional information can be gained from optic flow (Gibson, 1979; Horn, 1986). It is not known how this information is utilized by the brain in order to compute position. However, similar to the case of the vestibular sense, the brain has an easier task in computing angular selfmotion $v s$. linear self-motion. Assuming no large eye-movements, such as in the case of the rat, turning the head causes a uniform optic flow of the image on the retina. On the other hand,

\section{REFERENCES}

Beritoff, J. S. (1965). Neural Mechanisms of Higher Vertebrate Behavior. Boston: Little, Brown.

Blair, H. T. (1996). "A thalamocortical circuit for computing directional heading in the rat," in Advances in Neural Information Processing Systems, eds D. S. Touretzky, M. C. Moser, and M. E. Hasselmo (Cambridge: MIT press), 152-158.

Boccara, C. N., Sargolini, F., Thoresen, V. H., Solstad, T., Witter, M. P., Moser, E. I., and Moser, M.-B. (2010). Grid cells in pre- and parasubiculum. Nat. Neurosci. 13, 987-994.

Bonnevie, T., Fyhn, M., Hafting, T., Derdikman, D., Moser, E. I., and Moser, M. B. (2010). Hippocampal contribution to maintenance of entorhinal grid fields. Soc. Neurosci. Abstr. 101.4. San Diego, CA.

Bostock, E., Muller, R. U., and Kubie, J. L. (1991). Experience-dependent modifications of hippocampal place cell firing. Hippocampus 1, 193-205.

Brandon, M. P., Bogaard, A. R., Libby, C. P., Connerney, M. A., Gupta, K., and Hasselmo, M. E. (2011). Reduction of theta rhythm dissociates grid cell spatial periodicity from directional tuning. Science 332, 595-599.

Burgalossi, A., Herfst, L., von Heimendahl, M., Förste, H., Haskic, K., Schmidt, M., and Brecht, M. (2011). Microcircuits of

a linear change of head position has a more complex effect on optic flow: distant items move fast while close items move slowly. Furthermore, there is an ambiguity: a distant object moving fast can have a similar effect on optic flow as a proximal object moving slowly, and the brain needs some estimate of the distances of different objects in order to compute linear optic flow (Horn, 1986). To sum up, it is conceptually not surprising that different brain mechanisms and different sensory inputs have evolved in order to estimate spatial position $v s$. head direction, and that the two systems are anatomically separable, consistent with the findings in this paper.

\section{ACKNOWLEDGMENTS}

We thank Edvard Moser for reading and commenting on the manuscript, and May-Britt and Edvard Moser for giving their consent for the use of this data. We thank all of the people in the Kavli Institute for Systems Neuroscience and the Centre for the Biology of Memory in Trondheim for being both friendly and helpful all along. We thank the members of the Ulanovsky lab in the Weizmann Institute of Science for useful discussions. This work was supported by the Kavli Foundation and a Centre of Excellence grant from the Norwegian Research Council. Dori Derdikman is a David and Inez Myers Career Advancement Chair in Life Sciences fellow.

Chen, L. L., Lin, L.-H., Green, E. J., Barnes, C. A., and McNaughton, B. L. (1994). Head direction cells in the rat posterior cortex. Exp. Brain Res. $101,8-23$.

Cheng, K. (1986). A purely geometric module in the rat's spatial representation. Cognition 23, 149-178.

Clark, B. J., Bassett, J. P., Wang, S. S., and Taube, J. S. (2010). Impaired head direction cell representation in the anterodorsal thalamus after lesions of the retrosplenial cortex. J. Neurosci. 30, 5289-5302.

Clark, B. J., and Taube, J. S. (2011). Intact landmark control and angular path integration by head direction cells in the anterodorsal thalamus after lesions of the medial entorhinal cortex. Hippocampus 21, 767-782.

Derdikman, D., and Moser, E. I. (2010). A manifold of spatial maps in the brain. Trends Cogn. Sci. 14, 561-569.

Derdikman, D., Whitlock, J. R., Tsao, A., Fyhn, M., Hafting, T., Moser, M.-B., and Moser, E. I. (2009). Fragmentation of grid cell maps in a multicompartment environment. Nat. Neurosci. 12, 1325-1332.

Fuhs, M. C., and Touretzky, D. S. (2006). A spin glass model of path integration in rat medial entorhinal cortex. J. Neurosci. 26, 4266-4276.

Fyhn, M., Hafting, T., Treves, A., Moser, M. B., and Moser, E. I. (2007). Hippocampal remapping and grid realignment in entorhinal cortex. Nature 446, 190-194.

Gibson, J. J. (1979). The Ecological Approach to Visual Perception. Boston, MA: Houghton Mifflin.

Golob, E. J., and Taube, J. S. (1997). Head direction cells and episodic spatial information in rats without a hippocampus. Proc. Natl. Acad. Sci. U.S.A. 94, 7645-7650.

Goodridge, J. P., and Taube, J. S. (1995) Preferential use of the landmark navigatioal system by head direction cells in rats. Behav. Neurosci. 109 , 49-61.

Goodridge, J. P., and Taube, J. S. (1997). Interaction between the postsubiculum and anterior thalamus in the generation of head direction cell activity. J. Neurosci. 17, 9315-9330.

Hafting, T., Fyhn, M., Molden, S., Moser, M. B., and Moser, E. I. (2005). Microstructure of a spatial map in the entorhinal cortex. Nature 436, 801-806.

Horn, B. (1986). Robot Vision. Cambridge, MA: MIT Press.

Jezek, K., Henriksen, E. J., Treves, A., Moser, E. I., and Moser, M. B. (2011). Theta-paced flickering between place cell maps in the hippocampus. Nature 478, 246-249.

Jones, B. F., and Witter, M. P. (2007). Cingulate cortex projections to the parahippocampal region and hippocampal formation in the rat. Hippocampus 17, 957-976. 
Knierim, J. J., Kudrimoti, H. S., and McNaughton, B. L. (1995). Place cells, head direction cells, and the learning of landmark stability. J. Neurosci. 15, 1648-1659.

Knight, R., Hayman, R., Lin Ginzberg, L., and Jeffery, K. (2011). Geometric cues influence head direction cells only weakly in nondisoriented rats. J. Neurosci. 31, 15681-15692.

Koenig, J., Linder, A. N., Leutgeb, J. K., and Leutgeb, S. (2011). The spatial periodicity of grid cells is not sustained during reduced theta oscillations. Science 332, 592-595.

Langston, R. F., Ainge, J. A., Couey, J. J., Canto, C. B., Bjerknes, T. L., Witter, M. P., Moser, E. I., and Moser, M.B. (2010). Development of the spatial representation system in the rat. Science 328, 1576-1580.

Leutgeb, S., Leutgeb, J. K., Barnes, C. A., Moser, E. I., McNaughton, B. L., and Moser, M. B. (2005). Independent codes for spatial and episodic memory in hippocampal neuronal ensembles. Science 309, 619-623.

McNaughton, B. L., Barnes, C. A., Gerrard, J. L., Gothard, K., Jung, M. W., Knierim, J. J., Kudrimoti, H., Qin, Y., Skaggs, W. E., Suster, M., and Weaver, K. L. (1996). Deciphering the hippocampal polyglot: the hippocampus as a path integration system. J. Exp. Biol. 199, 173-185.

McNaughton, B. L., Battaglia, F. P., Jensen, O., Moser, E. I., and Moser, M. B. (2006). Path integration and the neural basis of the "cognitive map". Nat. Rev. Neurosci. 7, 663-678.

Mittelstaedt, M. L., and Mittelstaedt, H. (1980). Homing by path integration in a mammal. Naturwissenschaften 67, 566-567.

Muller, R. U., and Kubie, J. L. (1987). The effects of changes in the environment on the spatial firing of hippocampal complex-spike cells. J. Neurosci. 7, 1951-1968.

O’Keefe, J. (2007). “Hippocampal neurophysiology in the behaving animal," in The Hippocampus Book, eds P. Andersen, R. Morris, D. Amaral, T. Bliss, and J. O'Keefe (Oxford, NY: Oxford University Press), 475-540.

O’Keefe, J., and Dostrovsky, J. (1971). The hippocampus as a spatial map. Preliminary evidence from unit activity in the freely-moving rat. Brain Res. 34, 171-175.

O'Keefe, J., and Nadel, L. (1978). The Hippocampus as a Cognitive Map. Oxford, NY: Clarendon Press, Oxford University Press.

Ranck, J. B. (1985) "Head direction cells in the deep cell layer of dorsal presubiculum in freely moving rats," in Electrical Activity of the Archicortex, eds G. Buzsaki and C. H. Vanderwolf (Budapest: Akademiai Kiado), 217-220.

Samsonovich, A., and McNaughton, B. L. (1997). Path integration and cognitive mapping in a continuous attractor neural network model. J. Neurosci. 17, 5900-5920.

Sargolini, F., Fyhn, M., Hafting, T., McNaughton, B. L., Witter, M. P., Moser, M. B., and Moser, E. I. (2006). Conjunctive representation of position, direction, and velocity in entorhinal cortex. Science 312, 758-762.

Skaggs, W. E., Knierim, J. J., Kudrimoti, H. S., and McNaughton, B. L. (1995). A model of the neural basis of the rat's sense of direction. $A d v$. Neural Inf. Process. Syst. 7, 173-180.

Solstad, T., Boccara, C., Kropff, E., Moser, M. B., and Moser, E. I. (2008). Representation of geometric borders in the entorhinal cortex. Science 322, 1865-1868.

Stackman, R. W., Clark, A. S., and Taube, J. S. (2002). Hippocampal spatial representations require vestibular input. Hippocampus 12, 291-303.

Stackman, R. W., and Taube, J. S. (1997). Firing properties of head direction cells in the rat anterior thalamic nucleus: dependence on vestibular input. J. Neurosci. 17 4349-4358.

Stackman, R. W., Golob, E. J., Bassett, J. P., and Taube, J. S. (2003). Passive transport disrupts directional path integration by rat head direction cells. J. Neurophysiol. 90 2862-2874.

Taube, J. S. (1995). Head direction cells recorded in the anterior thalamic nuclei of freely moving rats. J. Neurosci. 15, 70-86.

Taube, J. S. (2007). The head direction signal: origins and sensory-motor integration. Annu. Rev. Neurosci. 30, 181-207.

Taube, J. S., and Burton, H. L. (1995). Head direction cell activity monitored in a novel environment and during a cue conflict situation. J. Neurophysiol. 74, 1953-1971.

Taube, J. S., Muller, R. U., and Ranck, J. B. (1990a). Head direction cells recorded from the postsubiculum in freely moving rats.1. Description and quantitativeanalysis. J. Neurosci. 10, 420-435.

Taube, J. S., Muller, R. U., and Ranck, J. B. (1990b). Head direction cells recorded from the postsubiculum in freely moving rats.2. Effects of environmental manipulations. J. Neurosci. 10, 436-447.

Tolman, E. C. (1948). Cognitive maps in rats and men. Psychol. Rev. 55, 189-208.

van Groen, T., and Michael Wyss, J. (1990). Connections of the retrosplenial granular a cortex in the rat. J. Comp. Neurol. 300, 593-606.

Whitlock, J. R., Derdikman, D., Pfuhl, G., Moser, M. B., and Moser, E.
I. (2010). Effects of parietal cortical inactivation on representations in entorhinal cortex. Soc. Neurosci. Abstr. 101.13. San Diego, CA.

Wills, T. J., Cacucci, F., Burgess, N., and O’Keefe, J. (2010). Development of the hippocampal cognitive map in preweanling rats. Science 328, 1573-1576.

Wills, T. J., Lever, C., Cacucci, F., Burgess, N., and O'Keefe, J. (2005). Attractor dynamics in the hippocampal representation of the local environment. Science 308, 873-876.

Yoder, R. M., Clark, B. J., Brown, J. E., Lamia, M. V., Valerio, S., Shinder, M. E., and Taube, J. S. (2011). Both visual and idiothetic cues contribute to head direction cell stability during navigation along complex routes. J. Neurophysiol. 105, 2989-3001.

Conflict of Interest Statement: The authors declare that the research was conducted in the absence of any commercial or financial relationships that could be construed as a potential conflict of interest.

Received: 22 November 2011; accepted: 22 February 2012; published online: 29 March 2012

Citation: Whitlock JR and Derdikman $D$ (2012) Head direction maps remain stable despite grid map fragmentation. Front. Neural Circuits 6:9. doi: 10.3389/ fncir.2012.00009

Copyright (c) 2012 Whitlock and Derdikman. This is an open-access article distributed under the terms of the Creative Commons Attribution Non Commercial License, which permits non-commercial use, distribution, and reproduction in other forums, provided the original authors and source are credited. 